http://jmscr.igmpublication.org/home/ ISSN (e)-2347-176x ISSN (p) 2455-0450 crossref DOI: https://dx.doi.org/10.18535/jmscr/v8i8.49

\title{
A Study on Mammography Findings on Clinically Palpable Breast Lesions and Assessing Its Accuracy by Comparing It with Histopathology
}

\author{
Authors \\ Deepa $S^{1}$, Beenamol $S^{2^{*}}$ \\ ${ }^{1}$ Assistant Professor, Department of Radiodiagnosis, Govt Medical College, Thiruvananthapuram \\ ${ }^{2}$ Additional Professor, Department of Radiodiagnosis, Govt Medical College, Thrissur \\ *Corresponding Author \\ Beenamol S
}

Additional Professor, Department of Radiodiagnosis, Government Medical College, Thiruvananthapuram,

Kerala, India

\begin{abstract}
Introduction: Incidence of breast cancer is on its rise. Breast cancer is the most common cancer among women in India. It is very important to recognise the lesion as benign or malignant. This is very important in deciding the treatment. Mammography is a commonly used imaging modality. The present study aims to find out the accuracy of mammography by comparing it with histopathology.

Materials and Methods: A descriptive study was conducted among 75 female patients above 20 years of age with palpable breast lesions. Patients with advanced carcinoma, history of previous breast biopsy and previously treated cancer are excluded from the study. Designed as diagnostic test evaluation, sensitivity, specificity, positive predictive value and negative predictive value of the variables were calculated comparing with histolopathological diagnosis. The features of the tests is analyzed by Cohen's Kappa for statistical agreement with histopathology.

Results: In this study, the overall sensitivity and specificity of mammography obtained are 92\%, and $87 \%$ respectively for differentiating benign from malignant breast lesions.

Conclusion: This study shows that mammography is useful in characterization of breast masses. Before going for biopsy, screening with mammography must be recommended.

Keywords: Benign, breast lesions, histopathology, malignant, mammograph.
\end{abstract}

\section{Introduction}

The incidence of cancer is on its rise in the present situation. There are several risk factors for a women to acquire breast cancer. Being female itself has got a higher risk. Age above fifty years, genetic mutations to BRCA1 and BRCA2 genes, longer exposure to hormones which include early menarche and delayed menopause, having dense breasts, history of breast cancer or certain noncancerous breast diseases, family history of breast cancer or any previous treatment using radiation therapy are non-modifiable risk factors. The modifiable risk factors include lack of exercise, sedentary life style, being less physically active, obesity, use of hormone replacement therapy or birth control pills, late first delivery and not breast 
feeding the baby. ${ }^{(1)}$ The most important thing in the case of a breast lump is to know whether it is benign or malignant as the treatment for either differs. The field of imaging is undergoing a rapid revolution due to invention of various new technologies. Screening helps to detect cancers at an earlier, more treatable stage. Improved technology in mammography units may decrease the radiation dose.

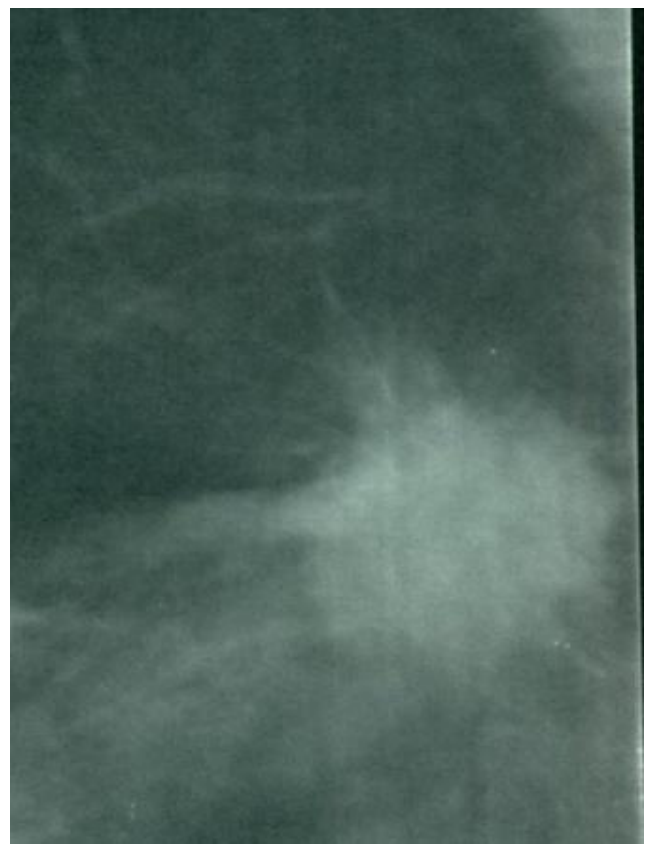

Figure1: Figure Spiculated borders seen in malignant breast lesions

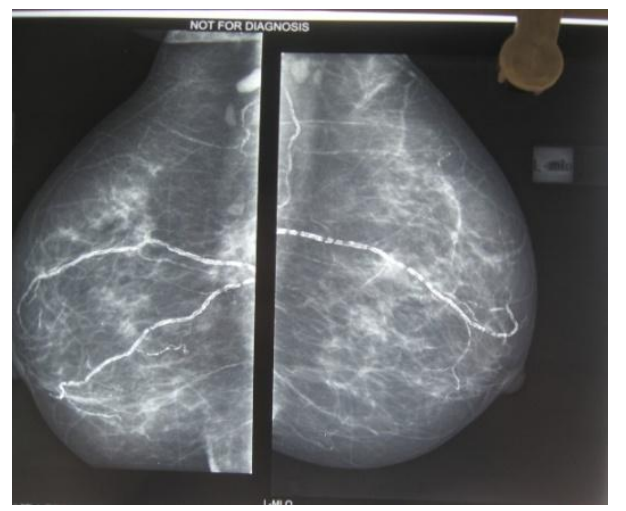

Figure 2: Mammogram showing vascular calcifications

Mammography is a radiographic examination or procedure that is designed for detecting breast pathology, particularly breast cancer. Mammography is the preferred method considered by radiologists, for the identification of breast calcifications. ${ }^{(2)}$ Mammography has find its usefulness in detecting conditions other than breast cancers such as breast abnormalities related to extra-mammary conditions such as congestive heart failure and central venous obstruction. ${ }^{(3)}$ Screening mammography is recommended every 1-2years for women once they reach 40 years of age and every year once they reach 50years of age. ${ }^{(4)}$ Dense breasts have connective tissue more compared to fat, which can sometimes make it hard to find tumors on a mammogram. Women with dense breasts are more prone to get breast cancer ${ }^{(1)}$. This has to be compared with the gold standard for confirmation. A very few studies have been done regarding mammographic findings of benign and malignant breast lesions and to assess its accuracy by comparing it with the findings of histopathology, which is the gold standard technique. This study is an attempt to evaluate the accuracy of mammography in characterizing breast lumps and comparing them with histopathology.

\section{Aim}

The aim of the study is to assess the mammographic findings of clinically palpable breast lesions for characterizing them as benign or malignant and to find out its accuracy by comparing with histopathology

\section{Objectives}

1. To assess the mammographic findings of clinically palpable breast lesions as benign or malignant

2. To assess the histopathological findings of clinically palpable breast lesions as benign or malignant

3. To determine the sensitivity, specificity, positive predictive value, negative predictive value and accuracy of mammography in differentiating benign versus malignant breast lesion with histopathological correlation.

\section{Material and Methods}

A descriptive study was conducted among female patients with breast lesions at the Department of 
Radiodiagnosis, Government Medical College, Thiruvananthapuram for one year. The study sample consisted of patients referred to Department of Radiodiagnosis for mammography to evaluate the palpable breast lesions.

All female patients above 20 years of age with palpable breast lesions were included for the study. Patients with advanced carcinoma (stage III and IV), previously treated breast cancer and those with history of breast biopsy were excluded from the study.

A structured questionnaire was used for data collection. The subjects were enrolled to the study by consecutive sampling method. There were 75 patients satisfying the inclusion criteria. After obtaining history, clinical examination was done. A written and informed consent was taken from them. The patients were subjected to mammography and then compared with histopathology. Histopathology is considered as the gold standard test. Thus, the accuracy of mammography can be determined.

\section{Data Collection}

Patients who satisfied the inclusion criteria were subjected to undergo mammography and confirmed the findings with FNAC or biopsy. Data collection was started after obtaining the Institutional Research and Ethical Committee Clearance. Low density, coarse calcifications and smooth margins are the benign criteria set, while, findings such as high density, micro calcifications, perifocal haziness and spiculated margins are considered as malignant criteria set for the present study.

\section{Data Analysis}

Data was analysed using SPSS 16.0. Frequency, percentage, sensitivity, specificity, positive predictive value, negative predictive value and accuracy of mammography for detection of benign and malignant breast lesions were assessed considering FNAC as the gold standard. It is derived from $2 \times 2$ tables with rows representing mammography positive and negative cases as well as columns representing FNAC positive and negative cases, which is the gold standard. The features of the tests were analyzed by Cohen's Kappa for statistical agreement between these and histopathology.

\section{Results}

The age of the patients ranged from 23 to 70 years. Majority belonged to 50-59 age group. About

$33.3 \%$ of them were in 50-59years age group, followed by $26.7 \%$ in $40-49$ years range and $18.7 \%$ in $30-39$ years range. $12 \%$ of the patients were of 60-69 years age group and 9.3\% in 20-29 years age group.

Table 1: Distribution of study subjects according to age

\begin{tabular}{|l|c|c|}
\hline Age in years & Frequency & Percentage $(\%)$ \\
\hline $20-29$ & 7 & 9.3 \\
\hline $30-39$ & 14 & 18.7 \\
\hline $40-49$ & 20 & 26.7 \\
\hline $50-59$ & 25 & 33.3 \\
\hline $60-69$ & 9 & 12 \\
\hline Total & $75(100)$ & $100(100)$ \\
\hline
\end{tabular}

Table 2: Distribution of study subjects according to clinical diagnosis

\begin{tabular}{|l|c|c|}
\hline Clinical diagnosis & Frequency & Percentage (\%) \\
\hline Benign & 27 & 36 \\
\hline Malignant & 28 & 37.3 \\
\hline Indeterminate & 20 & 26.7 \\
\hline
\end{tabular}


Table 3: Distribution of benign and malignant cases according to clinical diagnosis versus FNAC

\begin{tabular}{|l|c|c|c|c|c|c|}
\hline \multirow{3}{*}{ Clinical diagnosis } & \multicolumn{3}{|c|}{ FNAC findings } & \multicolumn{2}{c|}{ Total } \\
\cline { 2 - 7 } & \multicolumn{2}{|c|}{ Malignant } & \multicolumn{2}{c|}{ Benign } & \multicolumn{2}{c|}{} \\
\cline { 2 - 7 } & Frequency & Percentage & Frequency & Percentage & Frequency & Percentage \\
\hline Benign & 0 & 0 & 27 & 69.2 & 27 & 36 \\
\hline Malignant & 27 & 75 & 1 & 2.6 & 28 & 37.3 \\
\hline Indeterminate & 9 & 25 & 11 & 28.2 & 20 & 26.7 \\
\hline Total & 36 & 100 & 39 & 100 & 75 & 100 \\
\hline
\end{tabular}

Clinical diagnosis about type of malignancy and FNAC findings were found to be significant(p Value $<0.00)$

Table 4: Distribution of subjects according to the margin of lesion in mammography

\begin{tabular}{|l|c|c|}
\hline Margins & Frequency & Percentage(\%) \\
\hline Smooth & 29 & 38.7 \\
\hline Spiculated & 18 & 24 \\
\hline Irregular & 28 & 37.3 \\
\hline Total & 75 & 100 \\
\hline
\end{tabular}

Table 5: Distribution of benign and malignant cases according to margin

\begin{tabular}{|l|c|c|c|}
\hline Margins & Malignant $\mathrm{n}(\%)$ & Benign $\mathrm{n}(\%)$ & Total $\mathrm{n}(\%)$ \\
\hline Smooth & $1(2.8)$ & $28(71.8)$ & $29(38.7)$ \\
\hline Irregular & $8(22.2)$ & $10(25.6)$ & $18(24)$ \\
\hline Spiculated & $27(75)$ & $1(2.6)$ & $28(37.3)$ \\
\hline Total & $39(100)$ & $36(100)$ & $75(100)$ \\
\hline
\end{tabular}

Graph 1. Bar diagram showing distribution of benign and malignant subjects according to margin

Distribution of benign and malignant cases according to margin

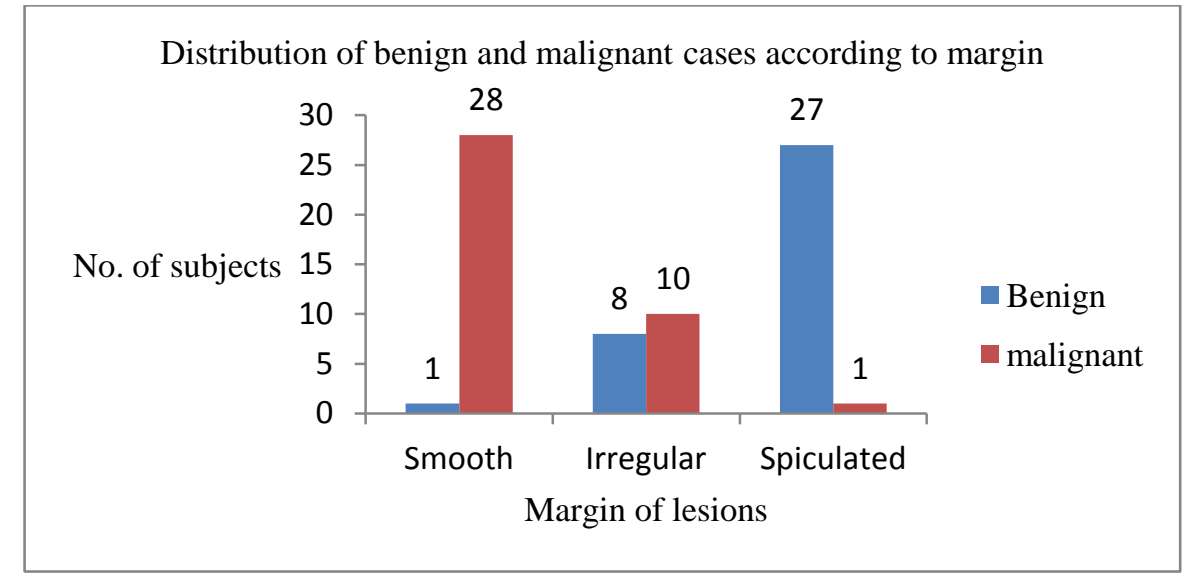

Table 6: Distribution of subjects according to Calcification

\begin{tabular}{|l|c|c|}
\hline Margins & Frequency & Percentage(\%) \\
\hline Nil & 25 & 33.3 \\
\hline Microcalcification & 23 & 30.7 \\
\hline Macrocalcification & 27 & 36 \\
\hline Total & 75 & 100 \\
\hline
\end{tabular}

Table 7: Distribution of subjects according to Histopathology

\begin{tabular}{|l|c|c|}
\hline Diagnosis & Frequency & Percentage(\%) \\
\hline Benign & 39 & 52 \\
\hline Malignant & 36 & 48 \\
\hline Total & 75 & 100 \\
\hline
\end{tabular}

Histopathology reports conclude that 39(52\% of the lesions) are benign and 36(48\%) are malignant. 
Table 8: Distribution of benign and malignant lesions according to type of calcification versus FNAC

\begin{tabular}{|l|c|c|c|c|c|c|}
\hline \multirow{2}{*}{ Calcification } & \multicolumn{3}{|c|}{ FNAC findings } & \multicolumn{2}{c|}{ Total } \\
\cline { 2 - 6 } & \multicolumn{2}{|c|}{ Malignant } & \multicolumn{2}{c|}{ Benign } & \multicolumn{2}{c|}{} \\
\cline { 2 - 6 } & Frequency & Percentage & Frequency & Percentage & Frequency & Percentage \\
\hline Nil & 12 & 33.3 & 13 & 33.3 & 25 & 33.3 \\
\hline Microcalcification & 23 & 63.9 & 0 & 0 & 23 & 30.7 \\
\hline Macrocalcification & 1 & 2.8 & 26 & 66.7 & 27 & 36 \\
\hline Total & 36 & 100 & 39 & 100 & 75 & 100 \\
\hline
\end{tabular}

The type of cancer into benign and malignant types based on calcification and FNAC findings were found to be significant $(\mathrm{p}<0.001)$.

Table 9 Distribution according to benign criteria and NPV of each characteristic in mammography

\begin{tabular}{|l|c|c|c|c|c|}
\hline \multirow{2}{*}{ Character } & \multicolumn{2}{|c|}{ FNAC } & Total n (\%) & Specificity & NPV \\
\cline { 2 - 3 } & Malignant n(\%) & Benign n(\%) & & & \\
\hline Low density & $2(5.6)$ & $16(41.0)$ & $18(24)$ & 41.0 & 66.7 \\
\hline Smooth margins & $1(2.8)$ & $28(71.8)$ & $29(38.7)$ & 71.8 & 72.4 \\
\hline Macrocalcifications & $1(2.8)$ & $26(66.7)$ & $27(36)$ & 66.7 & 72.2 \\
\hline
\end{tabular}

Table 10.Distribution according to malignant criteria and PPV of each characteristic in mammography

\begin{tabular}{|l|c|c|c|c|c|}
\hline \multirow{2}{*}{ Character } & \multicolumn{2}{|c|}{ FNAC } & Total & Sensitivity & PPV \\
\cline { 2 - 3 } & Malignant n(\%) & Benign n (\%) & n $\%$ & & \\
\hline High density & $34(94.4)$ & $23(59)$ & $57(76)$ & 94.4 & 59.6 \\
\hline Spiculated & $27(75)$ & $1(2.6)$ & $28(37.3)$ & 75.0 & 96.4 \\
\hline Macrocalcifications & $23(63.9)$ & $0(0)$ & $23(30.7)$ & 63.9 & 100.0 \\
\hline Perifocal haziness & $32(88.9)$ & $13(33.3)$ & $45(60)$ & 88.9 & 71.1 \\
\hline
\end{tabular}

Graph 2. Distribution of subjects to benign and malignant according to mammography and histopathology

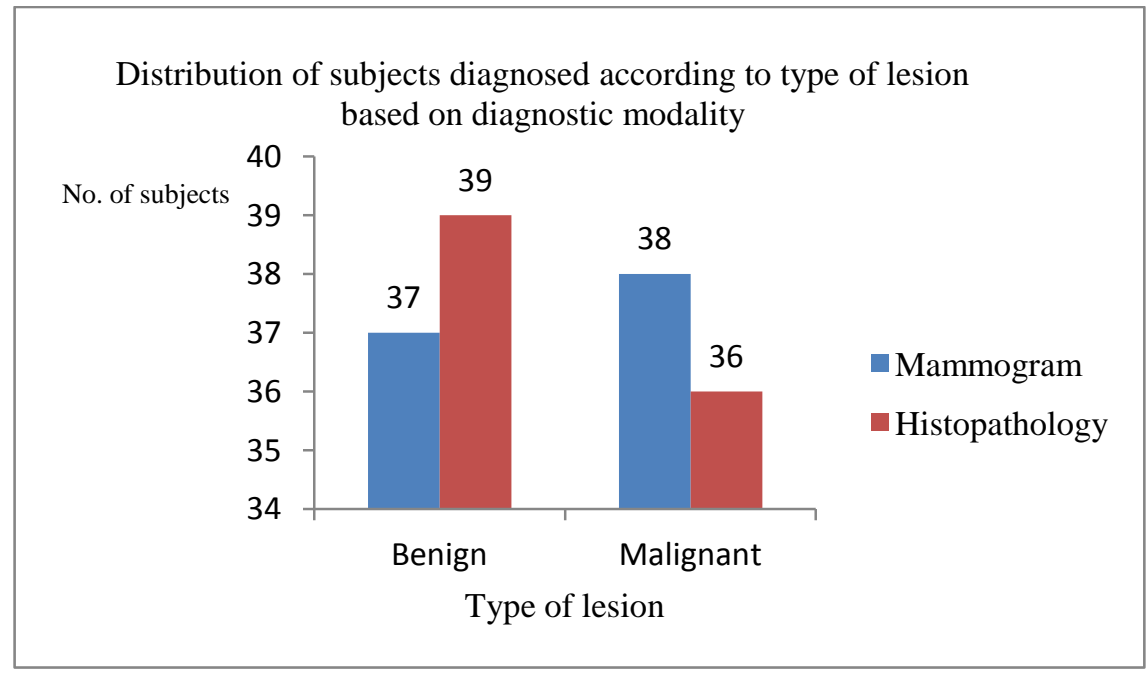

Table 11: Table showing Sensitivity, Specificity, PPV and NPV of mammography

\begin{tabular}{|l|c|c|c|}
\hline \multirow{2}{*}{ Mammographic diagnosis } & \multicolumn{2}{|c|}{ FNAC findings } & \multirow{2}{*}{ Total $\mathrm{n}(\%)$} \\
\cline { 2 - 3 } & Malignant $\mathrm{n}(\%)$ & Benign $\mathrm{n}(\%)$ & \\
\hline Malignant & $33(91.7)$ & $5(12.8)$ & $38(50.7)$ \\
\hline Benign & $3(8.3)$ & $34(87.2)$ & $37(49.3)$ \\
\hline Total & $36(100)$ & $39(100)$ & $75(100)$ \\
\hline
\end{tabular}

Measurement of agreement Kappa $=0.787, \mathrm{P}<0.001$

With mammography, the sensitivity, specificity and accuracy obtained were $91.6 \%, 87.1 \%$ and $89.33 \%$ respectively for detection of malignancy. PPV is $86.8 \%$ and NPV is $91.8 \%$. 


\section{Discussion}

Breast lump is very important matter of concern among women of any age and a diagnostic challenge to health care workers. The objective of the study was to assess the sensitivity, specificity and predictive values of mammography in differentiating benign and malignant breast lesions and to determine its accuracy by comparing with histopathology. The youngest patient with malignancy was 23 year old and the oldest patient was 67 year old. Above the age of 60 years, 4 out of 9 patients were malignant $(44.5 \%)$ and rest were benign cases which consisted of oil cysts, simple cysts and breast abscesses. In the present study, $44.5 \%$ in the age group above 60 years were malignant. There is higher chance of malignancy in a breast lump in older patients. According to histopathology, 39 lesions were benign and 36 lesions were malignant. Benign lesions that came across the study were simple cysts, breast abscess, galactocele and fibrocystic disease of the breast. According to histopathology, $48 \%$ of all the lesions were malignant and $52 \%$ were benign. These lesions were mammographically categorized as benign because they were low density lesions with smooth margins, that is satisfying the two benign criteria in mammography. According to mammography results, $49 \%$ were benign and $51 \%$ were malignant. According to mammography, five cases $(12.8 \%)$ were diagnosed as malignant and proved to be benign in histopathology. Three cases were having irregular margins and high density (satisfying 2 malignant criteria in mammography). Two cases were showing perifocal haziness with high density (satisfying 2 malignant criteria in mammography) and proved to be abscess in histopathology. Among the benign criteria for mammography $41 \%$ had low density. The low values in this study may be secondary to dense breast tissue in the respective cases. It is difficult to assess the character of lesion in mammography in patients with dense breasts (BIRADS 3 or 4 breast parenchyma). In this study, three cases which were considered as benign lesions in mammography turned out to be malignant in histopathology. Other benign criteria for mammography in our study were smooth margins and presence of macro calcification. Among the benign criteria, $72 \%$ of cases were having smooth margins on mammography with a NPV of $72.28 \%$ of patients with smooth margins on mammography were histologically proven as malignant. In the present study, microcalcifications are found in mammography. The correlation between mammographic and histopathologic findings are so comparable, to other studies where microcalcifications were the dominant finding, noted in $25(42 \%)$ of the 60 cases. ${ }^{(5)}$ In the present study, the sensitivity and specificity of mammography are $91.6 \%$ and $87.1 \%$ which is much better. In our study, the sensitivity and specificity of mammography were compared to other studies, and were calculated to be $73 \%$ and $55 \%$, and the rate of false negative was $17.27 \% .^{(6)}$ The mammography results were negative in $14.3 \%$ of patients because of high density of the breast. ${ }^{(7)}$ In this study, twenty three had microcalcification and all the study subjects who had microcalcifications turned to be malignant on histopathology, comparable with other studies also where microcalcification was the most frequent lesion reported among various studies and DCIS was also the common malignancy finding. ${ }^{(8)}$ The mammographic findings of invasive ductal carcinoma showed mass with microcalcifications and architectural distortion in 49 cases which is $48 \%$ of invasive ductal carcinoma cases. ${ }^{(9)}$

Those cases had other malignant features in mammography like calcifications and high density. Among the benign criteria for mammography $67 \%$ had macro calcifications and most of them were calcified fibroadenomas. The malignant criteria in mammography were high density, speculated margins, microcalcifications and perifocal haziness (if 2 or more criteria are satisfied it is taken as mammographically malignant). $64 \%$ of the cases showed micro calcifications. Positive predictive value of micro 
calcification is $100 \%$ as all the cases with this finding were malignant. Almost all (95\%) of malignant cases showed high density, $75 \%$ had spiculated margins and $89 \%$ had perifocal haziness. The positive predictive value of high density, spiculated margins and perifocal haziness were 60, 96, and 71 respectively. Spiculated margins have high positive predictive value for malignancy in mammography. With mammography, the sensitivity, specificity and accuracy obtained were $91.6 \%, 87.1 \%$ and $89.33 \%$ respectively for detection of malignancy. With sonomammography, the sensitivity, specificity and accuracy obtained were $83.3 \%$, $89.7 \%$ and $86.67 \%$ respectively for detection of malignancy. In this study the sensitivity is $92 \%$, specificity is $87 \%$ and positive predictive value is $87 \%$ of using mammography for differentiating benign and malignant masses and are comparable to other studies, where the sensitivity is $92 \%$, specificity is $94 \%$ and positive predictive value $84 \% .^{(10)}$ Another study showed that $22 \%$ of women who had palpable mass in the breast had false negative mammograms and concluded that biopsy should be performed in every undiagnosed, persistent mass without consideration of the mammogram. ${ }^{(11)}$ In a study of 201 cases of palpable solid breast masses, the sensitivity of clinical examination and mammography were $88 \%$ and $94 \%$ respectively. ${ }^{(12)}$ The mammographic sensitivity to detect carcinoma was $88.5 \%$. $(13,14)$ Another study where the palpable breast tumors and evaluated clinically and by mammography, then compared with the histopathology reports. Malignancy was confirmed in 51 patients and 60 were benign in HPR. The diagnostic accuracy for malignancy was $80.4 \%$ for mammography and $78.4 \%$ for clinical evaluation. The respective diagnostic accuracy for benign lesions was $85 \%$ and $91.7 \% .^{(15)}$

\section{Conclusion}

In this study, the overall sensitivity, specificity, positive and negative predictive values of mammography are calculated and it is found that mammography was more sensitive and specific in detecting malignant breast lesions. Those lesions with characteristic features of malignancy in mammography, like spiculated borders and microcalcifications with the branching pattern, can be considered as malignant and can be directly taken for surgery even without FNAC as these are more specific findings in malignancy and has a high positive predictive value. Together these imaging modalities can be reassuring if follow up is planned when the physical examination is not much suspicious and unnecessary breast biopsy can be avoided, which itself increases the risk. The kappa values for mammographic diagnosis were 0.787 with $\mathrm{p}$ value $<0.001$ which indicates good agreement with histopathology. It is very important as several biopsies can be avoided which itself can be a risk factor for breast cancer.

No sources of support in the form of grants

\section{References}

1. Ramchandra Kamath, Kamaleshwar S Mahajan, Lena Ashok, and T S Sanal. A study on risk factors of breast cancer among patients attending the tertiary care hospital in Udupi district. Indian $\mathbf{J}$ Community Med. 2013 Apr-Jun; 38(2): 95-99.

2. Bassett LW. Mammographic analysis of calcifications. Radiol Clin North Am. 1992 Jan;30(1):93-105.

3. Cao MM, Hoyt AC, Bassett LW. Mammographic signs of systemic disease. Radiographics. Jul-Aug;31(4):1085-100.

4. Schonberg M.A., Ramanan R.A., McCarthy E.P., Marcantonio E.R. Decision making and counseling around mammography screening for women aged 80 or older. J. Gen. Intern Med. 2006;21(9):979-985

5. Wei Tse Yang1,2 Gary M. K. Tse3 Sonographic, Mammographic, and Histopathologic Correlation of 
Symptomatic Ductal Carcinoma In Situ. AJR:182, January 2004; ;182:101-110

6. Qaed Salm Alhamami , Mohammed Hussain Almetlag, Malik Azhar Hussain , Ghadi Mohammed Al Hashan, Atheer Raja Alyami. The Egyptian Journal of Hospital Medicine (April 2018) Vol. 71 (2), 2603-2609 2603 Correlation of Ultrasound \& Mammography to Histopathology Results in Breast Cancer. A One Year Study at King Khalid Hospital, Najran, Saudi Arabia

7. Ghare khanloo F, Tarabian S, Kamrani S (2010): The study of the role of Additional ultrasound in diagnosis of breast cancer. J Hamadan Univ Med Sci Health Serv., 4(5): 60-7

8. GM Tse, Puay Hoon Tan, Amy LM Pang, Alice PY Tang, Humairah S Cheung. Classification in breast lesions: pathologists' perspective. Journal of Clinical Pathology. 2008;61(2):145-151. .

9. Chotiyano $\mathrm{A}^{1}, \quad$ Srinakarin $\mathrm{J}^{2}$, Triamwittayanont $T^{1}$, Wongsiri $T^{1}$, Koonmee $\mathrm{S}^{1}$ Asian archive of Pathology comparison of histopathology and mammography for breast lesions.

10. Yang WT, Mok CO, King W, Tang A, Metreweli C. Role of high frequency ultrasonography in the evaluation of palpable breast masses in Chinese women: alternative to mammography? J Ultrasound Med. 1996 Sep;15(9):637-44.

11. Edeiken S. Mammography and palpable cancer of the breast. Cancer. 1988 Jan 15;61(2):263-5.

12. van Dam PA, Van Goethem ML, Kersschot E, Vervliet J, Van den Veyver IB, De Schepper A, et al. Palpable solid breast masses: retrospective single- and multimodality evaluation of 201 lesions. Radiology. 1988 Feb;166(2):435-9.

13. Walsh P, Baddeley H, Timms H, Furnival CM. An assessment of ultrasound mammography as an additional investigation for the diagnosis of breast disease. $\mathrm{Br} \quad \mathrm{J}$ Radiol. 1985 Feb;58(686):115-9.

14. Guyer PB. Direct-contact B-scan sonomammography--an aid to X-ray mammography. Ultrasound Med Biol. 1988;14 Suppl 1:49-52.

15. De Gezelle H, Vanpeperstraete A, Defoort P, Serreyn R, Vandekerckhove D. Comparison of breast tumours evaluated by ultrasound, mammography, and clinical investigation. Arch Gynecol. 1981;230(3):219-23. 